\title{
Las calcificaciones del arco aórtico pueden predecir enfermedad coronaria y accidente cerebrovascular isquémico
}

Calcification of the Aortic Arch. Risk Factors and Association with Coronary Heart Disease, Stroke, and Peripheral Vascular Disease. Iribarren C, Sidney S, Sternfeld B et al. JAMA. 2000;283:2810-15

\section{Objetivo}

Evaluar factores de riesgo para la presencia de calcificaciones en el arco aórtico (CAA) y su asociación a largo plazo con enfermedades cardiovasculares.

\section{Diseño}

Estudio de cohorte prospectivo* poblacional

\section{Lugar}

Individuos pertenecientes a una sistema gerenciado de salud de California, EE.UU. (Kaisser Permanente).

\section{Pacientes}

El estudio incluyó 116309 afiliados (55 916 hombres y 60393 mujeres) entre 30 y 89 años de edad que participaron de exámenes periódicos de salud entre 1964 y 1973. La media de seguimiento fue de 26 años.

\section{Evaluación de los factores pronósticos}

Las variables pronósticas que se midieron fueron: edad, índice de masa corporal, colesterol sérico, nivel educacional, raza, hábito de fumar, consumo de alcohol, hipertensión, diabetes, historia familiar de infarto de miocardio y VDRL.

Medición del resultado principal

Se midió la tasa de hospitalizaciones o muerte por enfermedad coronaria, accidente cerebrovascular (ACV) isquémico, ACV hemorrágico y enfermedad vascular periférica, asociadas a la presencia de CAA en una telerradiografía de tórax de un examen periódico de salud. Las mismas fueron leídas por el grupo de radiólogos de Kaiser de acuerdo a las normas de la práctica diaria. Se registraron las hospitalizaciones y su diagnóstico al alta. Para las muertes se utilizó la base de datos del estado de California

\section{Resultados principales}

E $1,9 \%$ de los hombres y el $2,6 \%$ de las mujeres presentaron CAA. Su prevalencia se incrementó con la edad, en especial luego de los 65 años (10,6\% en los hombres y $15,9 \%$ en las mujeres). La edad mayor a 65 años, no tener educación secundaria, el tabaquismo y la hipertensión estuvieron independientemente asociados con las CAA en ambos sexos. En las mujeres, se asociaron también a la raza negra y a hiperlipemia. En cambio, las CAA estuvieron inversamente relacionadas al índice de masa corporal y a la historia familiar de infarto de miocardio.

En la tabla se observa la asociación de CAA y eventos vasculares. Los resultados están ajustados por edad, educación, raza, tabaquismo, consumo de atcohol, índice de masa corporal, colesterolemia, diabetes, hipertensión, e historia familiar de infarto de miocardio

\section{Conclusiones}

Las calcificaciones del arco aórtico, identificadas a través de una radiografía de tórax, estuvieron independientemente asociadas al riesgo de desarrollar enfermedad coronaria en ambos sexos, así como al riesgo de accidente cerebrovascular isquémico en las mujeres.

\section{Evento}

Galeificación del Areo Aórtico

\begin{tabular}{l|l}
\multirow{2}{*}{} & \multicolumn{1}{|c}{ Hombres } \\
\cline { 2 - 2 } & Riesgo Relativo \\
\hline Enfermedad Coronaria & 1,27 \\
\hline ACV isquémico & 1,17 \\
\hline ACV hemorrágico & 0,67 \\
\hline Enfermedad Vascular Periférica & 1,20 \\
\hline
\end{tabular}

(n 55 916
Mujeres (n 60 393) IC $95 \%$

\begin{tabular}{|l|l|}
\hline Riesgo Relativo \\
\hline 1,22 \\
\hline 1,46 \\
\hline 1,33 \\
\hline
\end{tabular}

\begin{tabular}{c}
\hline IC $95 \%$ \\
\hline 1,07 a 1,38 \\
\hline 1.28 a 1.67 \\
\hline 0,94 a 1,87 \\
\hline 0,97 a 1,39 \\
\hline
\end{tabular}

Fuente de financiamiento: no referida

\section{Comentario}

En los últimos años, múltiples son las publicaciones que se han referido a las implicancias de la presencia de depósitos cálcicos en la pared arterial y su papel en el desarrollo de eventos vasculares. Han prevalecido los estudios focalizados en el árbol coronario ${ }^{1}$, pero también se ha observado su rol en el contexto de la enfermedad cerebrovascular2.

En este estudio Iribarren y col. tratan de identificar por un lado, Ios factores de riesgo asociados al desarrollo de CAA, y por el otro, a la implicancia de éstas en el desarrollo de enfermedades cardiovasculares. La elección de un diseño de cohorte prospectivo* es adecuada para contestar esta pregunta. Las bases de datos en las que se basaron los diagnósticos de los eventos

finales habían sido validadas en estudios previos. Las posibles limitaciones del estudio, que no lo invalidan, son la baja sensibilidad de la placa de tórax para la detección de calcificaciones del árbol arterial comparada con otros métodos como la tomografía computada, y la ausencia de datos de calcificaciones en otras partes de la aorta.

Este estudio concluye que los pacientes con calcificaciones de arco aórtico presentan un mayor riesgo cardiovascular que los que los que no las tienen, y refuerza una vez más la importancia de la prevención primaria y secundaria en la práctica clínica diaria de los factores de riesgo para enfermedades cardiovasculares hasta hoy conocidos.

\section{Dr. Víctor Francisco Herrera [ Clínica Médica. ]}

Referencias

1. Wexler L, Brundage B, Crouse J et al.Coronary artery calcification: pathophysiology, epidemiology, imaging methods, and clinical implications: a statement for health professionals from the American Heart Association Writing Group. Circulation. 1996;94:1175-92

2. Cohen A., Tzourio C. Bertrand B, Chauvel C et al.Aortic plaque morphology and vascular events: a follow-up study in patients with isquemic stroke. Circulation. 1997;96:3838-41

Pregunta: El hallazgo de calcificaciones en el arco aórtico en una radiografía simple de torax sugiere que:

1. El paciente tiene cuatro veces más chance de desarrollar enfermedad coronaria

2. El paciente tiene entre y un 20 y un $25 \%$ veces más chance de desarrollar enfermedad coronaria

3. No tendrá enfermedad coronaria en los próximos 26 años

4. Si es varón es m'as probable que desarrolle enferemdad coronaria que enferemdad vascular periférica. 